\title{
04.1 \\ Возникновение усилий на клине при взаимодействии скользящего разряда со сверхзвуковым потоком
}

\author{
(C) В.М. Бочарников, В.В. Голуб, А.С. Савельев \\ Объединенный институт высоких температур РАН, Москва, Россия \\ E-mail: vova.m.bocha@gmail.com
}

Поступило в Редакцию 10 апреля 2020г.

В окончательной редакции 1 февраля 2021 г.

Принято к публикации 8 февраля 2021 г.

\begin{abstract}
Исследована сила, возникающая на клине при взаимодействии скользящего разряда со сверхзвуковым потоком. Скользящий разряд создавался в сверхзвуковой атмосферно-вакуумной аэродинамической трубе с числом Маха потока $\mathrm{M}=2$, числом Рейнольдса около $10^{6}$ и статическим давлением в потоке $p=0.15 \mathrm{bar}$. Установлено, что воздействие разряда не ограничивается появлением ударной волны, а также существенно меняет характер обтекания клина за счет импульсного тепловыделения. При увеличении энергии разряда эффект его воздействия на клин также увеличивается. Сила, создаваемая скользящим разрядом в сверхзвуковом потоке, в несколько раз больше, чем в неподвижном воздухе, независимо от величины давления: 0.15 или 1 bar.
\end{abstract}

Ключевые слова: скользящий разряд, сверхзвуковой поток, управление обтеканием.

DOI: 10.21883/PJTF.2021.09.50905.18334

Различные типы электрических разрядов могут быть использованы для управления сверхзвуковым обтеканием поверхности. Например, наносекундный диэлектрический барьерный разряд в потоке с числом Маха $\mathrm{M}=5$ создает ударную волну и действует как игла, смещающая падающую ударную волну на $25 \%$ вверх по течению [1]. Искровой разряд действует по-разному: быстрое выделение тепла изменяет течение в пограничном слое, что приводит к изменению распределения давления ниже по течению [2]. Искровой разряд занимает небольшой объем, поэтому для повышения эффективности его воздействия необходимо создавать массивы разрядников. Такие массивы могут быть либо линейными [3], либо распределенными по области [4]. Скользящий разряд лишен этого недостатка [5]. В настоящей работе в качестве активного элемента управления обтеканием использовался скользящий разряд по проводящей поверхности, инициируемый на клине. Упрощенно влияние разряда на поток можно представить следующим образом. При быстром локальном тепловыделении на поверхности клина в сверхзвуковом потоке посредством разряда газ нагревается до температуры в несколько тысяч градусов. В месте возникновения разряда образуется ударная волна, приводящая к перераспределению давления на поверхности профиля. При достаточной интенсивности ударной волны пограничный слой может отрываться от поверхности. В итоге результирующая сила действует на поверхность клина в течение определенного периода времени. Использование дугового скользящего разряда оправдано по следующим причинам. При инициировании обычного дугового разряда в воздухе необходимо приложить напряжение между электродами искрового промежутка, которое обеспечивает величину электрической напряженности, равную электрической прочности газа. Для атмосферного воздуха это значение составляет $30 \mathrm{kV} / \mathrm{cm}$. Если разряд инициируется в канале длиной $4 \mathrm{~cm}$, требуемое напряжение составляет $120 \mathrm{kV}$. При использовании скользящего разряда на проводящей поверхности эта величина составляет $2.5-5 \mathrm{kV}$, что соответствует уменьшению результирующего электрического поля более чем на порядок. В связи с тем, что объем газа, участвующего в скользящем разряде, увеличивается, доля энергии, выделяемой в газ в виде тепла, значительно увеличивается по сравнению с таковой в случае обычного дугового разряда [6,7]. Цель настоящей работы состоит в том, чтобы показать эффективность использования скользящего разряда для управления подъемной силой клина в сверхзвуковом потоке разреженного воздуха.

Работа проводилась на сверхзвуковой атмосферновакуумной аэродинамической трубе СТ-4 [8] (рис. 1). Установка состоит из сопла Лаваля, выполненного таким образом, чтобы придать прямоугольный профиль скорости потока в расчетном режиме, рабочей камеры с прозрачными оптическими стеклянными окнами для проведения видеосъемки движения модели, вакуумных резервуаров (газгольдеров) с общим объемом $240 \mathrm{~m}^{3}$, вакуумных насосов, откачивающих резервуары, и запорного устройства для запуска и остановки эксперимента. После откачки газгольдеров до давления менее $10 \mathrm{kPa}$ они заполняются воздухом через сопло, тем самым образуется сверхзвуковая струя внутри рабочей камеры, в которой размещается модель с разрядником. Основными параметрами воздушного потока являются число Маха $\mathrm{M}=2$, скорость потока $v_{0}=520 \mathrm{~m} / \mathrm{s}$, статическое давление $p_{s t}=0.15 \mathrm{bar}$, статическая температура $T_{s t}=160 \mathrm{~K}$. Диаметр выходного сечения сопла составляет $110 \mathrm{~mm}$. Для диагностики параметров потока использовались 


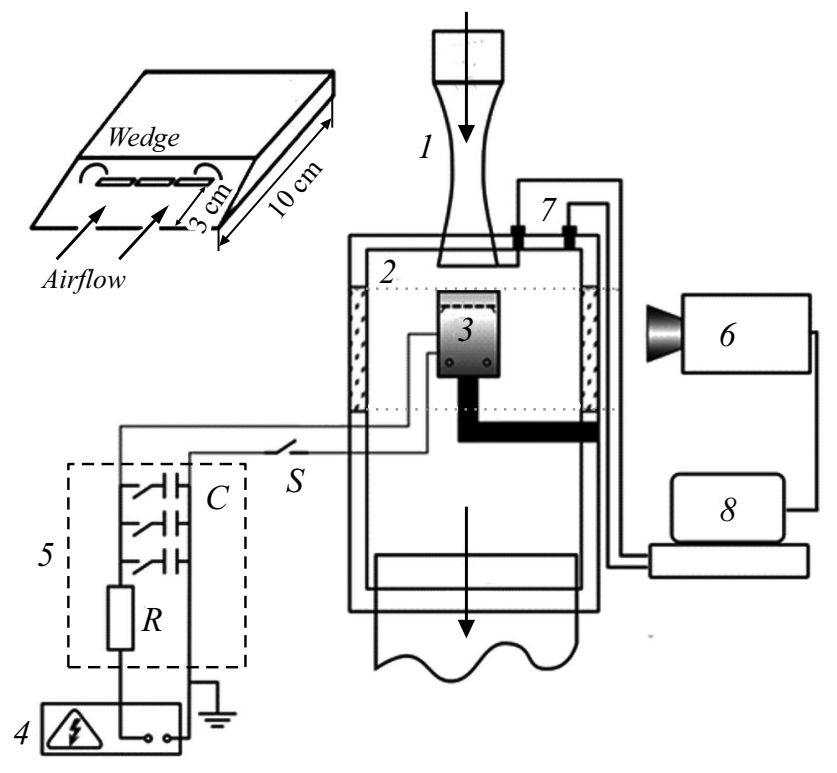

Рис. 1. Схема экспериментальной установки. 1 - сопло Лаваля, 2 - рабочая камера с прозрачными окнами, 3 - модель с разрядным устройством, 4 - высоковольтный источник питания, 5 - батарея конденсаторов, $6-$ высокоскоростная видеокамера, 7 - датчики давления, $8-$ компьютер для регистрации данных. На вставке - схема модели с разрядником. Стрелки указывают направление движения воздуха.

два датчика вакуумного давления Honeywell, один из которых был подключен к выходному сечению сопла и измерял статическое давление струи $p_{s t}$, второй был подключен к рабочей камере и измерял давление воздуха $p_{a}$, окружающего сверхзвуковой поток. Контроль давления необходим для проведения различных экспериментов с одинаковыми параметрами сверхзвуковой струи (при расчетном режиме течения, когда $p_{a}=p_{s t}$ ). В этом режиме течения с кромок сопла не сходят косые скачки уплотнения или волны разрежения, нарушающие однородность течения.

Для экспериментов использовалась клиновидная модель, на верхней поверхности которой располагалось разрядное устройство для создания протяженного искрового разряда в потоке (вставка на рис. 1). Разрядное устройство представляло собой сегментированный разрядник, где в качестве сегментов использовались три графитовых стержня прямоугольного сечения размером $11.3 \times 3.3 \times 3.3 \mathrm{~mm}$. Сегменты вмонтированы на поверхность модели заподлицо, а также отделены друг от друга диэлектрической пленкой (тефлоновая лента толщиной $0.15 \mathrm{~mm}$ ). Разрядное устройство располагалось на расстоянии $3 \mathrm{~cm}$ от передней кромки (вниз по потоку) модели в месте перегиба. Общая длина модели составляла $10 \mathrm{~cm}$. Максимальная толщина модели составляла $1 \mathrm{~cm}$. При замыкании ключа $S$ (рис. 1) и подаче высокого напряжения на выступающие проводящие электроды разрядного устройства по полупроводящей графитовой поверхности начинает бежать искра (стример), при этом разрядный ток усиливается многократно в момент, когда искра достигает противоположного электрода. Время этого процесса значительно меньше (менее $1 \mu \mathrm{s}$ ), чем пролетное время ( $100 \mu \mathrm{s})$. Важно отметить, что основной разряд (и выделение тепла) происходит в воздухе, который обтекает модель. Время основного разряда конденсаторов батареи составляло величину порядка пролетного времени. Угол при вершине клина составлял $10^{\circ}$, так что при обтекании его сверхзвуковым потоком воздуха не возникало отошедшей ударной волны. Размах модели составлял $8 \mathrm{~cm}$, что меньше поперечного размера сверхзвуковой струи. Модель закреплена не жестко относительно рабочей камеры. Это крепление (суппорт) позволяло смещаться модели на небольшое расстояние при придании ей небольшого импульса. Для определения импульса, приобретаемого моделью после разряда, выполнена калибровка суппорта. Для этого на поверхность модели в место, где происходит искровой разряд, свободно падал шар известной массы $m$ с высоты $L$ из пластичного материала, так что удар оказывался абсолютно неупругим. Масса $m$ гораздо меньше массы модели и суппорта. Регистрация смещения модели после падения шара производилась с помощью скоростной цифровой видеокамеры Photron FASTCAM SA-4. Съемка проводилась со скоростью $10000 \mathrm{fps}$ и экспозицией $2.6 \mu \mathrm{s}$. На полученных кадрах регистрировалось мгновенное положение модели. При максимальной величине отклонения клина от положения равновесия вся кинетическая энергия груза переходила в потенциальную энергию жесткости суппорта. Разделив кинетическую энергию груза перед ударом о клин на величину максимального отклонения модели от положения равновесия, можно получить среднее значение силы воздействия. В данном случае в расчет принималась сила, нормальная поверхности клина и ввиду малого угла его отклонения $\left(<1^{\circ}\right)$ вертикально направленная.

На рис. 2 представлена зависимость силы, рассчитанной по амплитуде колебаний клина, от энергии инициации скользящего разряда. Это суммарная сила

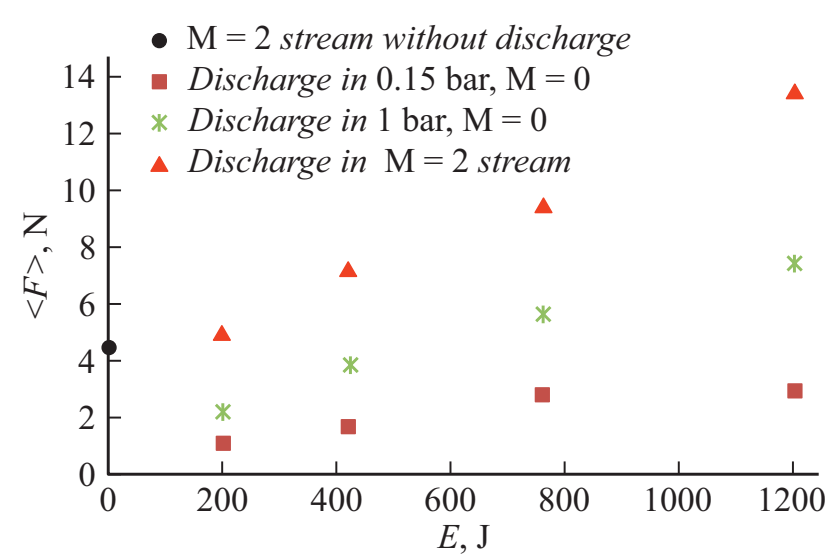

Рис. 2. Зависимость силы, возникающей от воздействия скользящего разряда на клин в потоке и без него, от энергии разряда. 


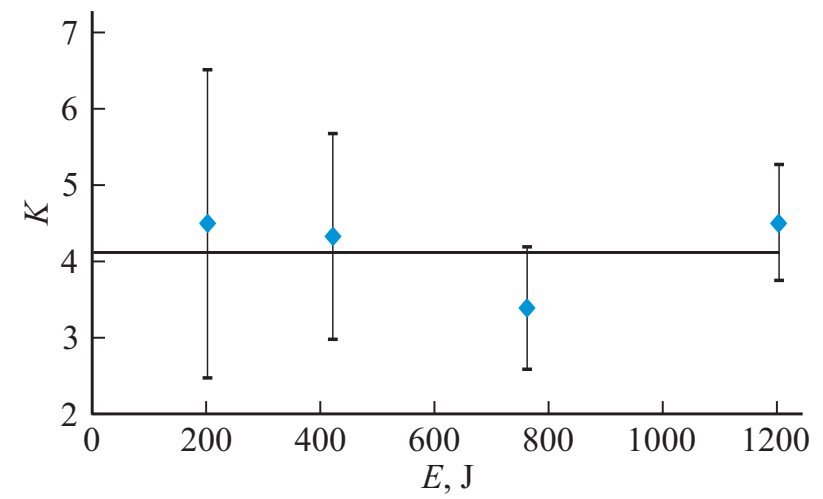

Рис. 3. Зависимость отношения силы, создаваемой скользящим разрядом в потоке и без него, от энергии инициирования скользящего разряда при давлении в камере 0.15 bar.

воздействия на клин, образованная действием ударной волны от скользящего разряда и эффектом изменения структуры сверхзвукового потока из-за быстрого тепловыделения. В данном случае эта сила регистрировалась по максимальному отклонению клина от положения равновесия после инициирования разряда, дальнейшие колебания с меньшей амплитудой не учитывались. Ввиду специфики измерения силы с усреднением абсолютной величины за четверть периода колебаний клина ( 1 ms) полученные значения следует интерпретировать в сравнении друг с другом для иллюстрации эффективности различных режимов взаимодействия клина с разрядом и потоком. Следует отметить четыре различных условия экспериментов. Сначала амплитуда колебаний клина в сверхзвуковом потоке измерялась без инициирования разряда. Средняя характерная сила $\langle F\rangle$ составляла $4 \mathrm{~N}$. Затем были проведены эксперименты по инициированию разряда без потока при давлениях 1 и $0.15 \mathrm{bar}$. В этом случае воздействие на клин осуществляется ударной волной, которая образуется в результате возникновения скользящего разряда. Как видно, с увеличением энергии инициирования мощность ударной волны также увеличивается. Однако средняя сила воздействия разряда превышает влияние потока только при высоких энергиях инициирования при атмосферном давлении. Завершающая серия экспериментов проводилась в сверхзвуковом потоке $\mathrm{M}=2$ при статическом давлении потока $0.15 \mathrm{bar}$. С ростом энергии инициирования разряда средняя сила значительно увеличивается и превышает как влияние потока, так и влияние ударной волны в его отсутствие. Исходя из этого можно сделать вывод, что эффект скользящего разряда не ограничивается созданием ударной волны, благодаря быстрому выделению тепла он также изменяет характеристики сверхзвукового воздушного потока вокруг клина, что приводит возникновению силы, которая значительно превосходит эффект ударной волны.

Следует также отметить, что при инициировании скользящего разряда в потоке пролетное время по порядку величины совпадает с длительностью разряда. Таким образом, тепловой эффект распространяется по всей длине клина, а не локализуется вблизи графитовых стержней. Таким образом, на верхней поверхности клина образуется область повышенной температуры, которая имеет максимум непосредственно возле разрядника и быстро убывает вниз по потоку. В первом приближении можно сказать, что данная область увеличивает давление на верхней поверхности клина за счет термодинамического эффекта в соответствии с уравнением Менделеева-Клапейрона. Оценка при энергии инициации $1200 \mathrm{~J}$ дает трехкратное увеличение давления, что в пересчете на силу по порядку величины соответствует полученным данным. Также следует упомянуть о том, что существование неоднородной области смешения в течение пролетного времени фактически изменяет геометрию клина и вносит существенные возмущения в сдвиговый слой, что приводит к кардинальной перестройке распределения давления по всей длине модели. На рис. 3 показана зависимость отношения $K$ воздействия разряда в потоке к его воздействию без потока при давлении 0.15 bar. Погрешности обусловлены тем, что колебания клина вследствие воздействия разряда накладываются на колебания клина под действием сверхзвукового потока. В пределах указанных погрешностей соотношение $K$ в потоке и без него не зависит от энергии инициирования в диапазоне от 200 до $1200 \mathrm{~J}$.

Во всех проведенных экспериментах скользящий разряд имел завершенную форму, те. энергия выделялась в объеме разрядного промежутка, а не в материале проводящей подложки. Такой режим существования разряда позволяет достичь максимальной эффективности и избежать разрушения электродов и проводящей подложки. Увеличение количества графитовых сегментов позволяет получить завершенную форму скользящего разряда при более низких значениях энергии инициирования. Таким образом, можно уменьшить энергию одиночного импульса и сделать процесс периодическим на частотах в десятки герц. Это позволит заменить однократное мощное воздействие постоянной силой, подверженной незначительным колебаниям под действием сверхзвукового потока.

В работе была исследована сила, возникающая на клине при взаимодействии скользящего разряда со сверхзвуковым потоком. Показано, что влияние скользящего разряда не ограничивается образованием ударной волны. Благодаря быстрому выделению тепла он изменяет сверхзвуковой поток воздуха вокруг клина, что приводит к созданию силы, значительно превосходящей эффект ударной волны. Сила, создаваемая скользящим разрядом в сверхзвуковом потоке, в несколько раз больше, чем в неподвижном воздухе, независимо от величины давления: 0.15 или 1 bar. По мере увеличения энергии разряда сила его воздействия на клин также увеличивается вплоть до $14 \mathrm{~N}$ при длине разрядного промежутка $4 \mathrm{~cm}$. Отношение силы, создаваемой скользящим разрядом в потоке и без него при давлении в 
камере $0.15 \mathrm{bar}$, не зависит от энергии инициирования разряда в диапазоне от 200 до $1200 \mathrm{~J}$.

\section{Конфликт интересов}

Авторы заявляют, что у них нет конфликта интересов.

\section{Список литературы}

[1] M. Nishihara, K. Takashima, J.W. Rich, I.V. Adamovich, Phys. Fluids, 23 (6), 066101 (2011).

https://doi.org/10.1063/1.3599697

[2] S.B. Leonov, I.V. Adamovich, V.R. Soloviev, Plasma Sources Sci. Technol., 25 (6), 063001 (2016).

[3] E. Caraballo, N. Webb, J. Little, J.H. Kim, M. Samimy, in 47th AIAA Aerospace Sciences Meeting including The New Horizons Forum and Aerospace Exposition (Orlando, Florida, 2009), p. AIAA 2009-924. https://doi.org/10.2514/6.2009-924

[4] T. Gan, Y. Wu, Z. Sun, D. Jin, H. Song, M. Jia, Phys. Fluids, 30 (5), 055107 (2018). https://doi.org/10.1063/1.5013166

[5] V.S. Aksenov, V.V. Golub, S.A. Gubin, A.S. Saveliev, V.A. Sechenov, E.E. Son, AIAA, 696, 5 (2009).

[6] V.F. Kopiev, Y.S. Akishev, I.V. Belyaev, N.K. Berezhetskaya, V.A. Bityurin, G.A. Faranosov, I.A. Moralev, J. Phys. D: Appl. Phys., 47 (50), 505201 (2014).

[7] Ф.Н. Глазырин, И.А. Знаменская, И.В. Мурсенкова, Д.С. Наумов, Н.Н. Сысоев, Письма в ЖТФ, 42 (1), 16 (2016).

[8] E. Son, Physical mechanics: laboratory workshops (Begell House Publ., 2016). 